\title{
COMPARATIVE ASSESSMENT OF DIFFERENT ENERGY MAPPING METHODS FOR GENERATION OF 511-KEV ATTENUATION MAP FROM CT IMAGES IN PET/CT SYSTEMS: A PHANTOM STUDY
}

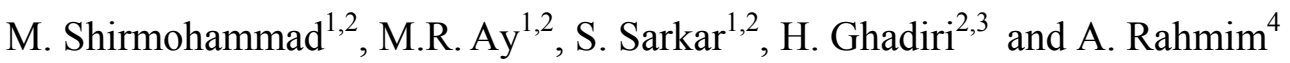 \\ ${ }^{1}$ Deptartment of Medical Physics and Biomedical Engineering, School of Medicine, Medical Sciences/ University \\ of Tehran, Tehran, Iran \\ ${ }^{2}$ Research Center for Science and Technology in Medicine, Medical Sciences/ University of Tehran, Tehran, Iran \\ ${ }^{3}$ Deptartment of Medical Physics, Iran University of Medical Science, Tehran, Iran \\ ${ }^{4}$ Department of Radiology, School of Medicine, Johns Hopkins University, Baltimore, USA.
}

\begin{abstract}
The use of X-ray CT images for CT-based attenuation correction (CTAC) of PET data results in the decrease of overall scanning time and creates a noise-free attenuation map ( $\mu$ map). The linear attenuation coefficient (LAC) measured with CT is calculated at the $\mathrm{x}$-ray energy rather than at the $511 \mathrm{keV}$. It is therefore necessary to convert the linear attenuation coefficients obtained from the CT scan to those corresponding to the $511 \mathrm{keV}$. Several conversion strategies have been developed including scaling, segmentation, hybrid, bilinear and dual-energy decomposition methods. The aim of this study is to compare the accuracy of different energy mapping methods for generation of attenuation map form CT images. An in-house made polyethylene phantom with different concentrations of K2HPO4 was used in order to quantitatively measure the accuracy of the nominated methods, using quantitative analysis of created $\mu$ maps. The generated $\mu$ maps using different methods compared with theoretical values calculated using XCOM cross section library. Accurate quantitative analysis showed that for low concentrations of K2HPO4 all these methods produce acceptable attenuation maps at $511 \mathrm{keV}$, but for high concentration of K2HPO4 the last three methods produced the lowest errors $(10.1 \%$ in hybrid, $9.8 \%$ in bilinear, and $4.7 \%$ in dual energy method). The results also showed that in dual energy method, combination of 80 and $140 \mathrm{kVps}$ produces the least error $(4.2 \%)$ compared to other combinations of $k V p s$.
\end{abstract}

Index Terms - CTAC, $\mu$ map, PET/CT, energy mapping

\section{INTRODUCTION}

The advent of PET/CT scanners is considered as a major advance in medical imaging technology and health care. PET/CT systems offer significant advantages over stand alone PET including decreased overall scanning time and increased accuracy in lesions localization [1]. One of the most important factors that affect both visual interpretation and quantitative accuracy of PET data is attenuation of gamma rays in tissues. It is well known that accurate attenuation correction is a vital component of PET imaging [2]. Reliable attenuation correction methods for PET require determination of an attenuation map, which represents the spatial distribution of linear attenuation coefficients at 511 $\mathrm{keV}$ for the region under study [3]. After the attenuation map is generated, it can be incorporated into image reconstruction algorithms to correct the emission data for errors contributed by photon attenuation. Attenuation correction in PET/CT scanners is based on using x-ray CT imaging. Generally, CT inherently provides a patientspecific measurement of the linear attenuation coefficient at each point in the image. However, the linear attenuation coefficient measured with $\mathrm{CT}$ is calculated at the $\mathrm{x}$-ray energy rather than at the $511 \mathrm{keV}$. It is therefore necessary to convert the linear attenuation coefficients obtained from the CT scan to those corresponding to the $511 \mathrm{keV}$. Several conversion strategies have been developed including scaling [4], segmentation [5], hybrid (segmentation/scaling) [6], bilinear [7], and dual-energy decomposition methods [8].

The limited number of publications assessing the influence of using different energy mapping methods on the accuracy of generated of attenuation map in CTAC method spurred the research presented in this paper. In this study, the accuracy of generated attenuation maps at $511 \mathrm{keV}$ using different energy mapping methods from the CT image of a dedicated phantom were assessed through comparison with measured attenuation coefficients at $511 \mathrm{keV}$ published by USA National Institute of Standards and Technology entitled as XCOM [9] cross section library.

\section{MATERIAL AND METHODS}

\section{Energy Mapping Methods:}

For CT based attenuation correction in PET/CT systems several approaches have been employed including scaling, segmentation, hybrid segmentation/scaling, bilinear, and dual-energy decomposition methods. In all of these methods attenuation correction requires accurate conversion from $\mathrm{CT}$ 
numbers to linear attenuation coefficients at PET energies (energy mapping), i.e. $511 \mathrm{keV}$. In the following, a short description of these methods is presented.

Scaling: In scaling method it is assumed that the ratio of the linear attenuation coefficient of a tissue at any two photon energies is essentially a constant. Attenuation image at $511 \mathrm{keV}$ is obtained by multiplying the CT image by the ratio of attenuation coefficients of water at $\mathrm{CT}$ and PET energies.

Segmentation: In this method attenuation image at 511 $\mathrm{keV}$ is formed by segmenting the reconstructed CT image into different tissue types. Then the CT numbers for each tissue type are replaced with the corresponding attenuation coefficients at $511 \mathrm{keV}$. Typical choices for tissue types are soft tissue, bone, and lung.

Hybrid: This method is based on a combination of the scaling and segmentation methods. The attenuation image at $511 \mathrm{keV}$ is formed by first using a threshold to separate out the bone component of the CT image, and then using separate scaling factors for the bone and non-bone component. This method is motivated by the fact that for most materials (an exception being bone), the ratio of the linear attenuation coefficients at any two photon energies is nearly a constant.

Bilinear: This model is based on the assumption that all tissues with $\mathrm{LAC}<\mathrm{LAC}_{\text {water }}$ are treated as a mixture of air and water at various concentrations and tissues with $\mathrm{LAC}>$ $\mathrm{LAC}_{\text {water }}$ are treated as a mixture of water and cortical bone. Since this approach results in the composition to have a single value for a $\mathrm{LAC}_{\mathrm{CT}}$; a bilinear scaling function is defined for biological tissues In this method, a series of CT scans from a known material (e.g. K2HPO4 solution) with different concentrations is performed. A calibration curve is then obtained in which the known attenuation coefficients at $511 \mathrm{keV}$ are plotted against the measured CT number. The resulting calibration curve is piece-wise linear. It should be noted that this is the most commonly used method in commercially available PET/CT scanners.

Dual-energy: A technically-challenging approach is to acquire the $\mathrm{CT}$ image at two different $\mathrm{kVps}$, and use these data to extract the individual photoelectric and Compton contributions to attenuation map. The different contributions can then be scaled separately in energy. This method is based on the fact that the Compton contribution decreases linearly with energy $\left(\mathrm{E}^{-1}\right)$ while the photoelectric contribution decreases rapidly as $\mathrm{E}^{-3}$. The two separate contributions can be scaled independently and combined to form the attenuation map at $511 \mathrm{keV}$. Dual-energy CTAC would allow for accurate attenuation correction in PET/CT imaging that involves high- $Z$ materials, including bone, contrast, and metals.

\section{Experimental Measurements:}

A polyethylene cylindrical phantom $(250 \pm 0.5 \mathrm{~mm}$ diameter) was constructed. This phantom consisted of 16 cylindrical holes $(20 \pm 0.5 \mathrm{~mm}$ diameter $)$ with four holes in the middle $(5 \pm 0.5 \mathrm{~mm}$ diameter $)$ which were filled with air. One of the 16 holes was filled with water and the rest of them with different concentrations of K2HPO4 in water (for modeling of soft tissue and bones with different densities). The concentrations K2HPO4 solution varied from $60 \mathrm{mg} / \mathrm{cc}$ to $1800 \mathrm{mg} / \mathrm{cc}$ to simulate bones with different densities.

This phantom was scanned by a LightSpeed VCT Volume CT scanner (GE Healthcare Milwaukee, USA) dedicated for cardiac imaging with four different tube voltages $(80,100,120$, and $140 \mathrm{kVp})$ and tube current of $400 \mathrm{~mA}$..

\section{Generation of umap and Comparison Strategy:}

The reconstructed CT images $(512 \times 512$ matrix size $)$ were down-sampled to $128 \times 128$ followed by Gaussian smoothing using a 5-mm kernel to match the resolution of the PET images. Then different energy mapping methods were used to convert CT pixel values in Hounsfield units (HU) to an attenuation map ( $\mu$ map) at $511 \mathrm{keV}$. The generated $\mu$ maps using each method were compared with the true value extracted from XCOM cross section library for different concentrations of K2HPO4. An ROI analysis was used for calculation of the relative errors between the values calculated from the attenuation maps generated using different methods and the true values for different concentrations of K2HPO4. For dual energy methods, different combinations of $\mathrm{kVps}(80,100,120$, and 140) with tube current of $400 \mathrm{~mA}$ were used and the $\mu$ maps at 511 $\mathrm{keV}$ was obtained for different combinations of $\mathrm{kVps}$.

\section{RESULTS}

Figure 1 shows the original CT image of the polyethylene phantom and the generated attenuation maps using different methods.

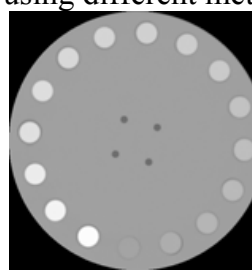

(a)

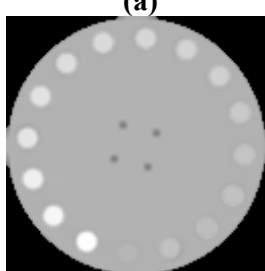

(d)

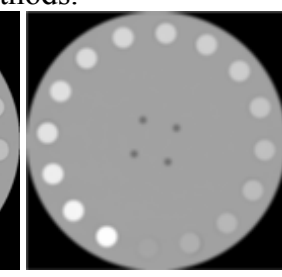

(b)

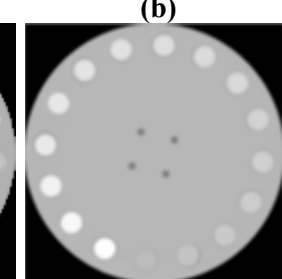

(e)

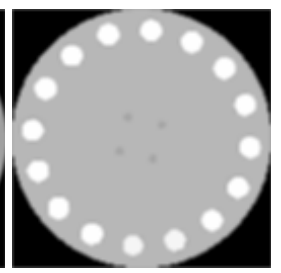

(c)

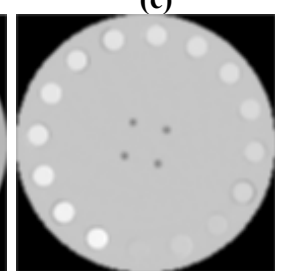

(f)
Figure 1: Original CT image (a) and generated attenuation maps using different methods, scaling (b), segmentation (c), hybrid (d), bilinear (e) and dual energy (100 and $120 \mathrm{kVp}$ ) (f).

Table 1 summarizes the relative percentage difference between the calculated linear attenuation coefficients at 511 $\mathrm{keV}$ for different regions of phantom with the true values extracted from the XCOM cross section library.

Table 1 
Relative percentage difference between the calculated LAC for different concentration of K2HPO4 with the reference values extracted from the XCOM cross section library.

\begin{tabular}{cccccc}
\hline Material Scaling & $\begin{array}{c}\text { Segment } \\
\text { ation }\end{array}$ & Hybrid & Bilinear & $\begin{array}{c}\text { Dual } \\
\text { Energy }\end{array}$ \\
\hline air & 0 & 0 & 0 & 0 & 0 \\
$\mathrm{H} 2 \mathrm{O}$ & 0 & 0 & 0 & 0 & 0.41 \\
$120^{\dagger}$ & 9.84 & 6.43 & 8.86 & 4.48 & 5.55 \\
180 & 18.00 & 2.73 & 0.09 & 9.14 & 7.63 \\
240 & 22.46 & 0.45 & 3.65 & 9.95 & 4.29 \\
300 & 25.44 & 3.36 & 6.20 & 11.34 & 3.63 \\
360 & 28.59 & 6.11 & 8.78 & 11.71 & 5.34 \\
480 & 32.43 & 11.38 & 12.19 & 13.41 & 4.30 \\
540 & 32.49 & 13.83 & 12.41 & 13.28 & 2.92 \\
600 & 34.28 & 16.02 & 14.02 & 13.55 & 3.00 \\
660 & 36.08 & 18.22 & 15.97 & 14.55 & 2.62 \\
720 & 37.08 & 20.26 & 16.53 & 14.55 & 2.12 \\
840 & 37.18 & 24.09 & 15.59 & 13.02 & 0.20 \\
900 & 36.14 & 25.95 & 16.30 & 12.63 & 0.88 \\
1200 & 34.08 & 33.65 & 14.85 & 9.06 & 6.57 \\
1500 & 28.05 & 40.04 & 10.06 & 3.46 & 12.76 \\
1800 & 23.83 & 45.30 & 6.07 & 2.65 & 17.26 \\
\hline
\end{tabular}

${ }^{\dagger} \mathrm{mgr} / \mathrm{cc}$ (Concentration of K2HPO4 in solution)

The average relative difference in all regions calculated using scaling, segmentation, hybrid, bilinear and dual energy methods are $27.25 \%, 16.74 \%, 10.10 \%, 9.8 \%, 4.97 \%$ respectively.

As there are several combinations of tube voltages for the dual energy method, it is useful to find out the optimum combination of tube voltages for CT imaging in order to generate more accurate attenuation map. In this study different combination of tube voltages were used for implementation of dual energy method. Figure 2 shows the generated attenuation map using dual energy method with different combination of $\mathrm{x}$-ray tube voltages.

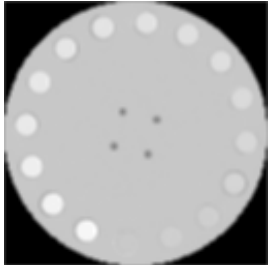

(a)

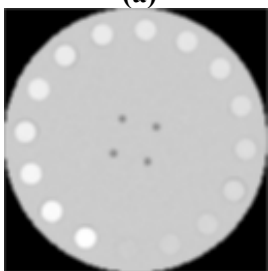

(d)

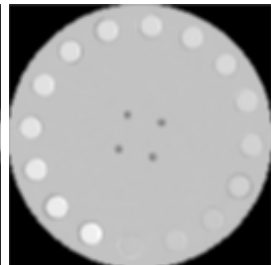

(b)

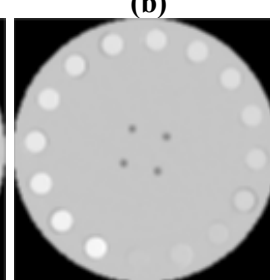

(e)

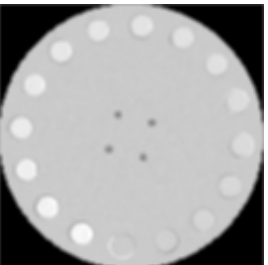

(c)

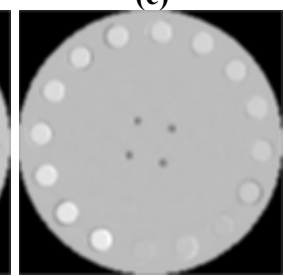

(f)
Figure 2. Generated attenuation map using dual energy method from the developed phantom with different combination of $\mathrm{x}$-ray tube voltages, $80-100 \mathrm{kVp} \mathrm{(a),} \mathrm{80-120} \mathrm{kVp} \mathrm{(b),} \mathrm{80-140} \mathrm{kVp} \mathrm{(c),}$ 100-120 kVp (d), 100-140 kVp (e) and 120-140 kVp (f).

Table 2 also shows the relative percentage difference of each combination of $\mathrm{kVps}$ compared to XCOM. It is obvious from this table that using 80 and $140 \mathrm{kVps}$ produces minimum difference.
Table 2

Relative percentage difference with the true value when using the dual energy method with different combination of $\mathrm{kVps}$.

\begin{tabular}{ccccccc} 
Material & $\begin{array}{c}\mathbf{8 0 - 1 0 0} \\
\mathbf{k V p}\end{array}$ & $\begin{array}{c}\mathbf{8 0 - 1 2 0} \\
\mathbf{k V p}\end{array}$ & $\begin{array}{c}\mathbf{8 0 - 1 4 0} \\
\mathbf{k V p}\end{array}$ & $\begin{array}{c}\mathbf{1 0 0 - 1 2 0} \\
\mathbf{k V p}\end{array}$ & $\begin{array}{c}\mathbf{1 0 0 - 1 4 0} \\
\mathbf{k V p}\end{array}$ & $\begin{array}{c}\mathbf{1 2 0 - 1 4 0} \\
\mathbf{k V p}\end{array}$ \\
\hline air & 0 & 0 & 0 & 0 & 0 & 0 \\
$\mathrm{H} 2 \mathrm{O}$ & 0.20 & 0.93 & 0.41 & 0.41 & 0.72 & 0 \\
$120^{\dagger}$ & 0.38 & 2.53 & 2.33 & 4.48 & 3.11 & 2.14 \\
180 & 3.29 & 2.54 & 1.22 & 7.63 & 2.82 & 2.26 \\
240 & 3.01 & 3.65 & 4.10 & 4.20 & 3.56 & 4.47 \\
300 & 3.90 & 3.72 & 4.07 & 3.63 & 4.16 & 4.16 \\
360 & 3.18 & 4.47 & 4.39 & 5.25 & 4.56 & 4.65 \\
480 & 1.86 & 3.17 & 3.41 & 4.06 & 3.57 & 3.82 \\
540 & 0.86 & 1.89 & 2.13 & 2.52 & 2.68 & 2.68 \\
600 & 0.15 & 1.15 & 1.77 & 2.46 & 2.31 & 2.61 \\
660 & 0.37 & 0.97 & 1.27 & 1.65 & 2.25 & 2.55 \\
720 & 1.24 & 0.65 & 0.58 & 1.02 & 1.09 & 0.87 \\
840 & 2.99 & 1.74 & 1.53 & 0.20 & 0.62 & 0.62 \\
900 & 4.55 & 3.12 & 2.58 & 0.88 & 1.83 & 2.17 \\
1200 & 11.13 & 9.00 & 7.85 & 6.57 & 6.81 & 6.81 \\
1500 & 16.33 & 14.2 & 13.75 & 12.7 & 12.87 & 12.3 \\
1800 & 21.02 & 19.1 & 18.51 & 17.2 & 17.6 & 17.6 \\
\hline$\dagger$ mgr/cc (Concentration of K2HPO4 in solution) &
\end{tabular}

The average relative difference in all regions calculated using dual energy method using different combination of tube voltages are shown in figure 3 . As it can be seen combination of 80 and $140 \mathrm{kVps}$ produces the minimum amount $(4.18 \%)$ of error among different combinations of $\mathrm{kVps}$.

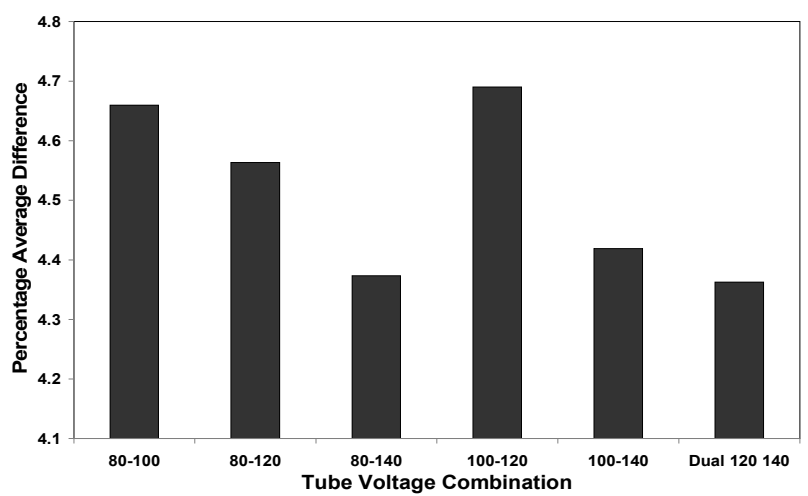

Figure 2. Average relative difference in all regions calculated using dual energy method with different combination of tube voltages.

\section{DISCUSSION}

As shown in table 1, all the methods yield acceptable results for water but for other regions which are presented by higher concentrations of K2HPO4, scaling and segmentation produce the highest errors. It was calculated from table 1 that the scaling method has the maximum amount of error $(27.25 \%)$ among other methods which increases for higher concentrations of K2HPO4. The high error of scaling is due to the fact that bones have a high effective atomic number hence increasing the contribution of photoelectric interaction in CT energies. Therefore scaling method overestimates the LAC at $511 \mathrm{keV}$. 
Although it seems that segmentation can be a reasonable method, it also has a high error. This is because in segmenting the CT image all the regions with CT numbers greater than $200 \mathrm{HU}$ are considered as bone and all of them are thus replaced with one LAC at $511 \mathrm{keV}$ corresponding to bone. The assigned LAC for bone at 511 $\mathrm{keV}$ is $0.109 \mathrm{~cm}^{-1}$ and so all the bones even with different densities (different concentrations of K2HPO4) will have a similar LAC at $511 \mathrm{keV}$. Considering the statements above and the data in table1, the bests methods would be hybrid (scaling/segmentation), bilinear and dual energy methods.

In the hybrid method a different scaling coefficient is used for bone (CT numbers greater than $200 \mathrm{HU}$ ) thus compensating the overestimation of LACs at $511 \mathrm{keV}$ yielded by scaling method. The scaling coefficient for bony regions of the phantom is the ratio of the $\mathrm{LAC}$ of bone at $\mathrm{CT}$ and PET energies.

According to table 1 the results of bilinear methods are in good agreement with theoretical values hence it is the commonly used method in most commercial PET/CT scanners.

Although the last three methods have the lowest errors in obtaining LACs at $511 \mathrm{keV}$, it seems that the best of these three is dual energy decomposition method. The relative error of this method compared to XCOM is $4.97 \%$. Some of the drawbacks of this method that render it impractical for commercial PET/CT scanners are the additional dose to the patient resulting from two CT scans of the patient at two different $\mathrm{kVps}$, increasing the scanning time as well as cost. However some groups have used special techniques to reduce the patient dose by CT scanning alternative slices with one of the $\mathrm{kVps}$. In addition since this was a phantom study, there was no restriction on dose. It should be noted that in the last two concentrations of K2HPO4 (1800 mg/cc and $1500 \mathrm{mg} / \mathrm{cc}$ ), this method has a relative higher error compared to other concentrations and this is due to the fact that the effective atomic numbers of the last two concentrations are so high that the contribution of photoelectric interaction increases regarding to it.

In this study different combinations of operational $\mathrm{kVps}$ were used in order to assess and compare the resultant $\mu$ maps for each combination of $\mathrm{kVps}$. The results summarized in table 2, show that the more is the difference between $\mathrm{kVps}$, the lowest would be the errors in resultant $\mu$ maps, i.e. selecting 80 and $140 \mathrm{kVps}$ is the best option. In performing dual energy method, the effective energy of CT spectrum for each operational $\mathrm{kVps}$ was required. The effective energy of each $\mathrm{kVp}$ was calculated according to the method proposed by Bai et al. . According to these calculations for $\mathrm{kVps}$ of $80,100,120$, and 140 the effective energy was obtained to be $56 \mathrm{keV}, 63 \mathrm{keV}, 70 \mathrm{keV}$, and 76 $\mathrm{keV}$, respectively.

\section{CONCLUSION}

This phantom study demonstrated that hybrid (scaling/segmentation), bilinear and dual energy methods yield acceptable $511 \mathrm{keV}$ mmaps particularly dual energy method produces the lowest error, hence in conditions where there is not any restriction on patient dose (similar to the phantom study here) this method can be the best option. In addition a better plan would be to further extend these investigations into clinical settings with a more realistic phantom or actual patients in order to use the results in clinical arena. Another result of this study is that when utilizing the dual energy method, selecting the dual $\mathrm{kVps}$ with the maximum difference yields the best resultant $\mu$ map at $511 \mathrm{keV}$. It appears beneficial and probable that the dual energy method will be the method of choice for energy mapping in future generations of PET/CT scanner equipped with high definition X-ray tubes, with the possibility of dual energy imaging in one scan without increasing patient dose using ultra fast switching of $\mathrm{x}$-ray tube voltage between two different $\mathrm{kVps}$.

\section{REFERENCES}

[1] D. W. Townsend, J. P. J. Carney, J. T. Yap, and N. C. Hall, "PET/CT today and tomorrow.," J Nucl Med, vol. 45, pp. 4S-14S, 2004.

[2] M. R. Ay and H. Zaidi, "Assessment of errors caused by x-ray scatter and use of contrast medium when using CT-based attenuation correction in PET.," Eur J Nucl Med Mol Imaging, vol. 33, pp. 1301-1313, 2006.

[3] M. R. Ay and H. Zaidi, "Computed Tomography-based attenuation correction in neurological positron emission tomography: evaluation of the effect of x-ray tube voltage on quantitative analysis.," Nucl Med Commun, vol. 27, pp. 339-346, 2006.

[4] T. Beyer, P. E. Kinaham, D. W. Townsend, and D. Sashin, "The use of X-ray CT for attenuation correction of PET data," in Proc. IEEE Nuclear Science Symposium and Medical Imaging Conference., Rome, Italy, 1995, pp. 1573 - 1577.

[5] P. E. Kinahan, B. H. Hasegawa, and T. Beyer, "X-ray-based attenuation correction for positron emission tomography/computed tomography scanners.," Semin Nucl Med, vol. 33, pp. 166-179, 2003.

[6] P. E. Kinahan, D. W. Townsend, T. Beyer, and D. Sashin, "Attenuation correction for a combined 3D PET/CT scanner.," Med Phys, vol. 25, pp. 2046-2053, 1998.

[7] C. Bai, L. Shao, A. J. Da Silva, and Z. Zhao, "A generalized model for the conversion from CT numbers to linear attenuation coefficients.," IEEE Trans Nucl Sci, vol. 50, pp. 1510-1515, 2003.

[8] M. J. Guy, I. A. Castellano-Smith, M. A. Flower, G. D. Flux, R. J. Ott, and D. Visvikis, "DETECT-dual energy transmission estimation CT-for improved attenuation correction in SPECT and PET.," IEEE Trans Nucl Sci, vol. 45, pp. 1261 -1267, 1998.

[9] M. J. Berger, J. H. Hubbell, S. M. Seltzer, J. Chang, J. S. Coursey, R. Sukumar, and D. S. Zucker, "XCOM: photon cross sections database. NBSIR 87-3597." vol. 2005 Gaithersburg: Ionizing Radiation Division, Physics Laboratory, National Institute of Standards and Technology, Gaithersburg, MD 20899 NIST, Physics Laboratory, Office of Electronic Commerce in Scientific and Engineering Data, 1998. 